DrAFt VERSION July 5, 2021

Preprint typeset using $\mathrm{L}^{A} \mathrm{~T}_{\mathrm{E}} \mathrm{X}$ style emulateapj v. 08/22/09

\title{
GRBS AND HYPERNOVA EXPLOSIONS OF SOME GALACTIC SOURCES
}

Gerald E. Brown

Department of Physics and Astronomy, State University of New York, Stony Brook, NY 11794, USA.

Chang-Hwan Lee

Department of Physics, Pusan National University, Busan 609-735, Korea.

AND

EnRIQUe Moreno MÉndez

Department of Physics and Astronomy, State University of New York, Stony Brook, NY 11794, USA.

Draft version July 5, 2021

\begin{abstract}
Knowing the Kerr parameters we can make quantitative calculations of the rotational energy of black holes. We show that Nova Sco (GRO J1655-40), Il Lupi (4U 1543-47), XTE J1550-564 and GS $2023+338$ are relics of gamma-ray burst (GRB) and Hypernova explosions. They had more than enough rotational energy to power themselves. In fact, they had so much energy that they would have disrupted the accretion disk of the black hole that powered them by the communicated rotational energy, so that the energy delivery was self limiting. The most important feature in producing high rotational energy in the binary is low donor (secondary star) mass.

We suggest that V4641 Sgr (XTE J1819-254) and GRS 1915+105 underwent less energetic explosions; because of their large donor masses. These explosions were one or two orders of magnitude lower in energy than that of Nova Sco. Cyg X-1 (1956+350) had an even less energetic explosion, because of an even larger donor mass.

We find that in the evolution of the soft X-ray transient sources the donor (secondary star) is tidally locked with the helium star, which evolved from the giant, as the hydrogen envelope is stripped off in common envelope evolution. The tidal locking is transferred from the helium star to the black hole into which it falls. Depending on the mass of the donor, the black hole can be spun up to the angular momentum necessary to power the GRB and Hypernova explosion. The donor decouples, acting as a passive witness to the explosion which, for the given angular momentum, then proceeds as in the Woosley Collapsar model.

High mass donors which tend to follow from low metallicity give long GRBs because their lower energy can be accepted by the central engine.

Subject headings: binaries: close — gamma rays: bursts — black hole physics — supernovae: general - X-rays: binaries
\end{abstract}

\section{INTRODUCTION}

The hypernova explosions accompanying GRBs are Type $\mathrm{I}_{b c}$; i.e., they show no hydrogen lines and no helium lines. Arguments have been given that the helium lines would not be seen even if the helium were present, that helium would have to mix with ${ }^{56} \mathrm{Ni}$ if the lines were to be seen, etc. Thus, hydrogen is not present at the time of the explosion. As we shall outline, this is the situation in common envelope evolution in Case $\mathrm{C}$ mass transfer. Case $\mathrm{C}$ mass transfer means mass transfer after the helium burning of the giant is finished. For such a case the GRB and hypernova explosion for Nova Sco (GRO J1655-40) was described by Brown et al. (2000). We can now reconstruct the explosion for this case, since the Kerr parameters $\left(a_{\star}\right)$ of Nova Sco and Il Lupi have been measured in the Smithsonian-Harvard-MIT observations (Shafee et al. 2006), with $a_{\star}=0.65-0.75$ and $a_{\star}=0.75-0.85$ respectively. They check against the prediction of Lee et al. (2002) (denoted as LBW) who found

Electronic address: GEB: gbrown@insti.physics.sunysb.edu

Electronic address: CHL: clee@pusan.ac.kr

Electronic address: EMM: moreno@grad.physics.sunysb.edu $a_{\star}=0.8$ for both. From the $a_{\star}$ we can construct available energies in the Blandford-Znajek mechanism. We have a simple guiding principle for the sources considered; namely, that the explosion energy depends chiefly on the mass of the donor (secondary star), and this is easily seen if the binaries are evolved in Case $\mathrm{C}$ mass transfer, as we shall show.

In similar vein, the GRBs and hypernova explosions can be constructed for XTE J1550-564 and GS 2023+338 (V404 Cygni), the available rotational energy being nearly the same as in Nova Sco.

Moreno Méndez et al. (2007) also reconstructed the explosions of GRS $1915+105$ and V4641 Sgr. They found the explosion of Cyg X-1, in agreement with Mirabel \& Rodrigues (2003), to be a dark explosion; i.e., orders of magnitude less explosive than Nova Sco.

\section{ROLE OF DONOR STAR IN COMMON ENVELOPE EVOLUTION}

Using the relation between the $\mathrm{He}$ core mass $\left(M_{\mathrm{He}}\right)$ of a giant after finishing H-core burning and the initial giant mass $\left(M_{\text {giant }}\right)$,

$$
M_{\mathrm{He}}=0.08\left(M_{\text {giant }} / M_{\odot}\right)^{1.45} M_{\odot},
$$


LBW found that following common envelope evolution,

$$
a_{f} \simeq \frac{M_{d}}{M_{\odot}}\left(\frac{M_{\text {giant }}}{M_{\odot}}\right)^{-0.55} a_{i} .
$$

Here $a_{f}$ is the final separation of the He star which remains from the giant following the strip off of its $\mathrm{H}$ envelope, and $a_{i}$ is its initial separation, $M_{d}$ is the mass of the donor (secondary star). Noteworthy about eq. (2) is that the main dependence of the final separation $a_{f}$ is on the donor mass $M_{d}$, only roughly as the square root of $M_{\text {giant }}$.

The He star remainder of the giant and the donor are tidally locked at the end of common envelope evolution (LBW). The tidal locking ends here, the Kerr parameter of the black hole being determined by its angular momentum at formation, minus the decrease from angular momentum spent in powering explosions.

From Kepler we have for the preexplosion period

$$
\frac{\text { days }}{P_{b}}=\left(\frac{4.2 R_{\odot}}{a_{f}}\right)^{3 / 2}\left(\frac{M_{d}+M_{\mathrm{He}}}{M_{\odot}}\right)^{1 / 2}
$$

where $M_{d}$ and $M_{\mathrm{He}}$ are the masses at the time of common envelope evolution. Given $P_{b}$ we can easily find the Kerr parameter $a_{\star}$ from Fig. 12 of LBW, reproduced as Figure 1 here. In the case of Nova Sco, $P_{b}=1 / 4$ day, $a_{f}=5.33 R_{\odot}, M_{\mathrm{He}}=11 M_{\odot}$ and $M_{d}=1.91 M_{\odot}(\mathrm{LBW})$.

The big advantage that Case $\mathrm{C}$ mass transfer has is that it not only produces an explosion with no hydrogen envelope, but it produces a great deal of angular momentum, as quantified in the Kerr parameter of the black hole, to power the GRB and Hypernova. The angular momentum results from the tidal locking of the donor and the He star, the latter falling into the black hole. In the core, the helium is burned before common envelope evolution into carbon and, rather quickly, oxygen. The strong $\vec{B}$-field lines, which at one end thread the disk of the black hole as it is formed from the collapse inwards of the ionized metals, are frozen at the other end in the metals and lock the disk tidally with these metals which constitute what is left of the original helium star. If we replace the helium star in the MacFadyen \& Woosley (1999) paper by our He star then the formation and spin up of the black hole is as these authors described. Thus we basically have a collapsar with high angular momentum that has been spun up by tidal locking with the donor. Note that there is no hydrogen envelope of the giant left, the hydrogen having been expelled in common envelope evolution.

\section{GRBS AND HYPERNOVAE FROM SOFT X-RAY} TRANSIENTS WITH EVOLVED COMPANIONS

In Table 1 we list the black hole masses, and our estimates of donor masses, all at the time of the end of common envelope evolution when the tidal locking was established between donor and helium star. These came from LBW and from Moreno Méndez et al. (2007). The Kerr parameters are changed into energies using the Blandford-Znajek formulas (Lee et al. 2000)

$$
E_{B Z}=1.8 \times 10^{54} \epsilon_{\Omega} f\left(a_{\star}\right) \frac{M_{B H}}{M_{\odot}} \operatorname{ergs}
$$

where the efficiency $\epsilon_{\Omega}=\Omega_{F} / \Omega_{H}$ for energy deposition in the (perturbative) fireball is taken to be $1 / 2$ (for op- timum impedance matching) and

$$
f\left(a_{\star}\right)=1-\sqrt{\frac{1}{2}\left(1+\sqrt{1-a_{\star}^{2}}\right) .}
$$

We note that Cyg X-1 $(1956+350 y)$ probably went through a dark explosion (Mirabel \& Rodrigues 2003) meaning that at most, a very low energy, one or two magnitudes less than in the case of Nova Sco. The high Kerr parameter $\left(a_{\star}>0.98\right)$ for GRS 1915-105 (McClintock et al. 2006) came chiefly from mass accretion following the explosion in which the black hole was born, and, therefore, had no influence on the GRB (Moreno Méndez et al. 2007). The measured Kerr parameters are the present ones, and the energies to produce the GRB and Hypernova should be subtracted from our calculated ones.

What we see from Table 1 is that the transient sources Nova Sco, Il Lupi, XTE J1550-564, and GS 2023+338 clearly had enough rotational energy to power both a GRB and Hypernova explosion. Brown et al. (2000) in discussing these for Nova Sco suggested that the energy was so great that the explosion disrupts the accretion disk; this removes the magnetic fields anchored in the disk and self-limits the energy the BlandfordZnajek mechanism can deliver (see the appendix). In addition to the 7 sources in Table 1 LBW worked out the Kerr parameters of the 8 Galactic X-ray Transient sources with main sequence companions, all of which had $a_{\star}$ 's of $0.6-0.8$ which correspond to spin energies of $430-600 \times 10^{51}$ ergs.

In Brown et al. (2000) the GRB and hypernova explosion were reconstructed in all detail. The F-star donor in Nova Sco bore witness to the hypernova explosion through the $\alpha$-particle nuclei deposited on it. In particular, a large amount of Sulfur, which Nomoto et al. (2000) found typical of differentiating hypernovae from the more usual supernovae, was found. The Kerr parameter of 0.8 found by LBW for the preexplosion spin was, within uncertainties, the same as the post explosion Kerr parameters measured by Shafee et al. (2006). The GRB was, of course, not recorded, but the rotational energy was tremendous so that the GRB was either just begun or the accretion disk was smashed immediately. The system velocity was worked out. Almost all of the natal angular momentum energy is still in the system, as measured by Shafee et al. (2006), meaning that very little was accepted for the explosion.

\section{SUBLUMINOUS GRBS}

In Brown et al. (2000) the population synthesis suggested a soft X-ray transient birth rate of $3 \times 10^{-4}$ sources per year per galaxy, which with $10^{5}$ galaxies within 200 Mpc translates into $3750 \mathrm{Gpc}^{-3} \mathrm{yr}^{-1}$. If we consider the beaming factor of $\sim 10 \%$, this is the same rate as the rate of subluminous sources investigated by Liang et al. (2007), estimated at $325_{-177}^{+352} \mathrm{Gpc}^{-3} \mathrm{yr}^{-1}$. The latter are thought to have come from low-metallicity galaxies, but it is none the less interesting that the rate of hypernovae from soft X-ray transient sources is the same as that of the subluminous bursts, especially because we have shown that only a small part of the black hole spin energy in soft X-ray transient sources went into the explosion, so that they would tend to be subluminous. 
The question of central engine for GRB060218 was tackled by the 119 astronomers who signed the 5 papers in Nature (Campana et al. 2006; Mazzali et al. 2006; Pian et al. 2006; Soderberg et al. 2006; Young 2006). From the Supplementary Information of Mazzali et al. (2006) one finds that the explosion 2006aj was Type $\mathrm{I}_{b c d}$ in nature; i.e., in addition to no hydrogen lines, no helium nor carbon. The only place where this could occur was in a black hole in which convective carbon burning ceases because the carbon abundance drops below $15 \%$ : see Fig. 1 of Brown et al. (2001). This leaves no doubt but that the central engine was powered by a black hole, one of low mass.

Galactic GRBs (GRBs from galaxies with solar metallicity) must be subluminous, relatively little of their tremendous rotational energy being used up in the explosion. For the population of metal poor subluminous GRBs one would expect their donors to be more massive because of their low metallicity. Because of the more massive donors they will have less rotational energy, which may be all utilized in the explosions or, at least, will take larger to dismantle the disk. Thus they would be of relatively long duration, but subluminous in the integrated energy in the explosion.

Recently an eclipsing binary M33 X-7 was discovered in a metal poor neighborhood ( $\sim 10 \%$ solar $)$ by Orosz (2007). This can be evolved like a more massive Cyg X1 , but with the advantage that one knows the donor to be $\sim 80 M_{\odot}$ at the time of explosion. The Kerr parameter was $a_{\star}=0.12$ and the angular momentum energy of $\sim 10^{52}$ ergs was too little to both power the jet for a GRB and the hypernova, so the explosion was probably "dark." Had the donor been less massive, according to our arguments, then with more energy the GRB and hypernova could have been powered. We agree that the subluminous bursts come chiefly from metal poor galaxies (Stanek et al. 2006), giving the dynamical reason that they have low angular momentum energies because of larger donor masses.

\section{DISCUSSION}

We show that the rotational energy of black holes in soft X-ray transient sources is greatest when the donor in the binary is of low mass. In the case of large donor masses, the rotational energy in the black hole binary is lower.

One can see that Nova Sco had a very high explosion energy from the fact that its space velocity after the explosion is $112 \pm 18 \mathrm{~km} \mathrm{~s}^{-1}$ as to compare with Cyg X-1 relative to Cyg OB3 in the cluster of O-stars of $9 \pm 2 \mathrm{~km}$ $\mathrm{s}^{-1}$, which is typical of the random velocities of stars in expanding associations (Mirabel \& Rodrigues 2003).

The explanation of why the angular momentum energy is so high in Nova Sco was given on p.176 of Bethe et al. (2003): "The massive star will have evolved through its supergiant (He core burning stage) before matter overflows its Roche lobe. Then, by that time, a main sequence companion must be at just the right distance to receive the overflow; this means $a \sim 1500 R_{\odot}$, the Roche lobe of the massive star being at $\sim \frac{2}{3} a$. Since the binding energy of the envelope of the massive star goes as $1 / a$, this binding energy is very small, so that the envelope can be removed by the drop in gravitational energy of an $\sim 1 M_{\odot}$ main sequence star as it moves inwards in common envelope evolution with the massive star from $\sim 1500 R_{\odot}$ to the much smaller Roche lobe of the He star which results when the $H$ envelope is removed from the massive star. In this way one could understand why all of the main sequence companions of the black holes in the transient sources were of nearly the same low masses, $(0.5-1) M_{\odot} . "$ For the companions with masses $10 M_{\odot}$, the necessary drop in gravitational energy is only $1 / 10$ that of the $1 M_{\odot}$ companion, so the final $a_{f}$ can be an order of magnitude greater. The result is an order of magnitude lower rotational energy.

From the above explanation we see that the ultrahigh rotational energies in soft X-ray transient sources are a result of the low donor masses. The rotational energy drops roughly inversely with mass so we would expect it to be an order of magnitude less for stars of low metallicity whose masses are roughly an order of magnitude greater than stars in our Galaxy. Thus, cosmological GRBs will not have so much rotational energy as to dismantle the disk, and may be able to furnish their rotational energy to the GRB and Hypernova. At least, now that we understand why Galactic GRBs are so energetic, we can offer reasons why the cosmological GRBs have lower energy, but may be able to use up more of it in the explosion.

Measurement of the Kerr parameter for XTE J1550564 (J. McClintock et al., Smithsonian-Harvard coalition, in progress) will enable us to say how much of the natal $\sim$ 300Bethes was used up in the explosion.

\section{SUMMARY}

In summary, the essential points of our paper are that the Woosley Collapsar model can be obtained from our Case $\mathrm{C}$ mass transfer, but with the black hole having any desired angular momentum, by making choice of the donor mass. Because the helium is burned preceding the explosion in Case $\mathrm{C}$ mass transfer, the ashes of the central helium, carbon and oxygen, fall first into the black hole and ensure the tidal locking through the strong B-field lines which are frozen in the ionized metals.

Our results for the LBW calculation of Kerr parameters have been confirmed by the Smithsonian-Harvard group. Given the Kerr parameters, we can make quantitative calculation of the spin energy of black hole. We give predictions for the Kerr parameters of 12 Galactic black hole sources which have not yet been measured.

We note that the rotational energy of M33 X-7 was lower than that of cosmological GRBs and suggest that these originate from low metallicity donors of somewhat less mass than that of M33 X-7. Our suggestion that XTE J1550-564 should have the angular momentum energy in its explosion, which is as large as that of cosmological GRBs, should soon be tested by the measurement of the Kerr parameter.

\section{ACKNOWLEDGMENTS}

We would like to thank Jeff McClintock for many useful discussions. G.E.B. was supported by the US Department of Energy under Grant No. DE-FG02-88ER40388. C.H.L. was supported by Grant No. R01-2005-00010334-0 (2005) from the Basic Research Program of the Korea Science \& Engineering Foundation. 


\section{APPENDIX}

DISMANTLING THE ACCRETION DISK BY HIGH ENERGY INPUT

Knowing the Kerr parameters we can make quantitative estimates of energy. The amount of energy poured into the accretion disk of the black hole, and, therefore, also pressure is almost unfathomable., the $5 \times 10^{53}$ ergs being 500 times the energy of a strong supernova explosion, the latter being spread over a much larger volume than that of the accretion disk. Near the horizon of the black hole, the physical situation might become quite complicated (Thorne et al. 1986). Field-line reconstruction might be common and lead to serious breakdowns in the freezing of the field to the plasma; and the field on the black hole sometimes might become so strong as to push its back off the black hole and into the disk (Rayleigh-Taylor Instability) concentrating the energy even more. During the instability the magnetic field lines will be distributed randomly in "globs", the large ones having eaten the small ones. It seems reasonable that the Blandford-Znajek mechanism is dismantled. Later, however, conservation laws demand that the angular momentum not used up in the GRB and hypernova explosion be reconstituted in the Kerr parameter of the black hole.

\section{REFERENCES}

Bethe, H.A., Brown, G.E., and Lee, C.-H. 2003, Formation and Evolution of Black Holes in the Galaxy : Selected Papers with Commentary, World Scientific Series in 20th Century Physics Vol. 33 (World Scientific, 2003).

Brown, G.E., Heger, A., Langer, N., Lee, C.-H., Wellstein, W., and Bethe, H.A. 2001, New Astronomy, 6, 457.

Brown, G.E., Lee, C.-H., Wijers, R.A.M.J. Lee, H.K., Israelian, G. and Bethe, H.A. 2000, New Astronomy 5, 191.

Campana, S. et al. 2006, Nature 442, 1008.

Cantiello, M., Yoon, S.-C., Langer, N., and Livio, M. 2007, A\&A 465, L29.

Lee, H.K., Wijers, R.A.M.J., Brown, G.E. 2000, Physics Reports $325,83$.

Lee, C.-H., Brown, G.E., and Wijers, R.A.M.J. 2002, ApJ, 575, 996 (LBW).

Liang, E., Zhang, B., Virgili, F., and Dai, Z.G. 2007, ApJ, 662, 1111.

MacFadyen, A.I. and Woosley, S.E. 1999 ApJ 524, 262.

MacFadyen, A.I., Woosley, S.E., and Heger, A. 2001, ApJ, $550,410$.

Mazzali, P.A. et al. 2006, Nature 442, 1018.
McClintock, J.E., Shafee, R., Narayan, R., Remillard, R.A., Davis, S.W., and Li, L.-X. 2006, ApJ, 652, 518.

Mirabel, I.F., Rodrigues, I. 2003, Science, 300, 1119.

Moreno Méndez, E., Brown, G.E., Lee, C.-H. and Walter, F. 2007 , in progress.

Nomoto et al., The Greatest Explosions since the Big Bang: Supernovae and Gamma-Ray Bursts, CUP:Cambridge; astro-ph/0003077

Orosz, J. et al. 2007, Nature, 449, 872.

Pian, E. et al. 2006, Nature 442, 1011.

Shafee, R., McClintock, J.E., Narayan, R., Davis, S.W., Li, L., and Remillard, R.A. 2006, ApJ, 636, L113.

Soderberg, A.M. et al. 2006, Nature 442, 1014.

Sollerman, J., Östlin, G., Fynbo, J.P.U., Hjorth, J., Fruchter, A., and Pedersen, K. 2005, New Astronomy, 11, 103.

Stanek, K.Z. et al. 2006, Acta Astronomica, 56, 333.

Thorne, K.S., Price, R.H. and Macdonald, A., Black Holes: The Membrane Paradigm New Haven and London, 1986, p.133.

Young, T.R. 2006, Nature 442, 992. 


\begin{tabular}{|c|c|c|c|c|}
\hline Name & $\begin{array}{c}M_{B H} \\
{\left[M_{\odot}\right]}\end{array}$ & $\begin{array}{c}M_{d} \\
{\left[M_{\odot}\right]}\end{array}$ & $a_{\star}$ & $\begin{array}{c}E_{\mathrm{BZ}} \\
{[\text { Bethes }]}\end{array}$ \\
\hline GRO J1655-40 & $\sim 5$ & $1-2$ & 0.8 & $\sim 430$ \\
4U 1543-47 & $\sim 5$ & $1-2$ & 0.8 & $\sim 430$ \\
\hline XTE J1550-564 & $\sim 10$ & $1-2$ & 0.5 & $\sim 300$ \\
GS 2023+338 & $\sim 10$ & $1-2$ & 0.5 & $\sim 300$ \\
\hline XTE J1819-254 & $6-7$ & $\sim 10$ & 0.2 & $10 \sim 12$ \\
GRS 1915+105 & $6-7$ & $\sim 10$ & $0.2\left(>0.98^{\dagger}\right)$ & $10 \sim 12$ \\
Cyg X-1 & $6-7$ & $\gtrsim 30$ & 0.15 & $5 \sim 6$ \\
\hline
\end{tabular}

TABLE 1

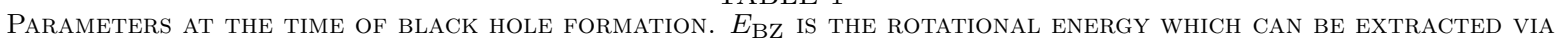
BLANDFORD-ZNAJEK MECHANiSM With OPTIMAL EFFICIENCY $\epsilon_{\Omega}=1 / 2$ IN EQ. (4), EXCEPT FOR LOW $a_{\star} \mathrm{S}, a_{\star}=0.2$ AND 0.15 WHERE THE efficiencies are lower, give $\epsilon_{\Omega}=0.37$ and 0.33 as Calculated in Appendix 2 of Brown et al. (2000). ${ }^{\dagger}$ Kerr parameter is the PRESENT ONE.

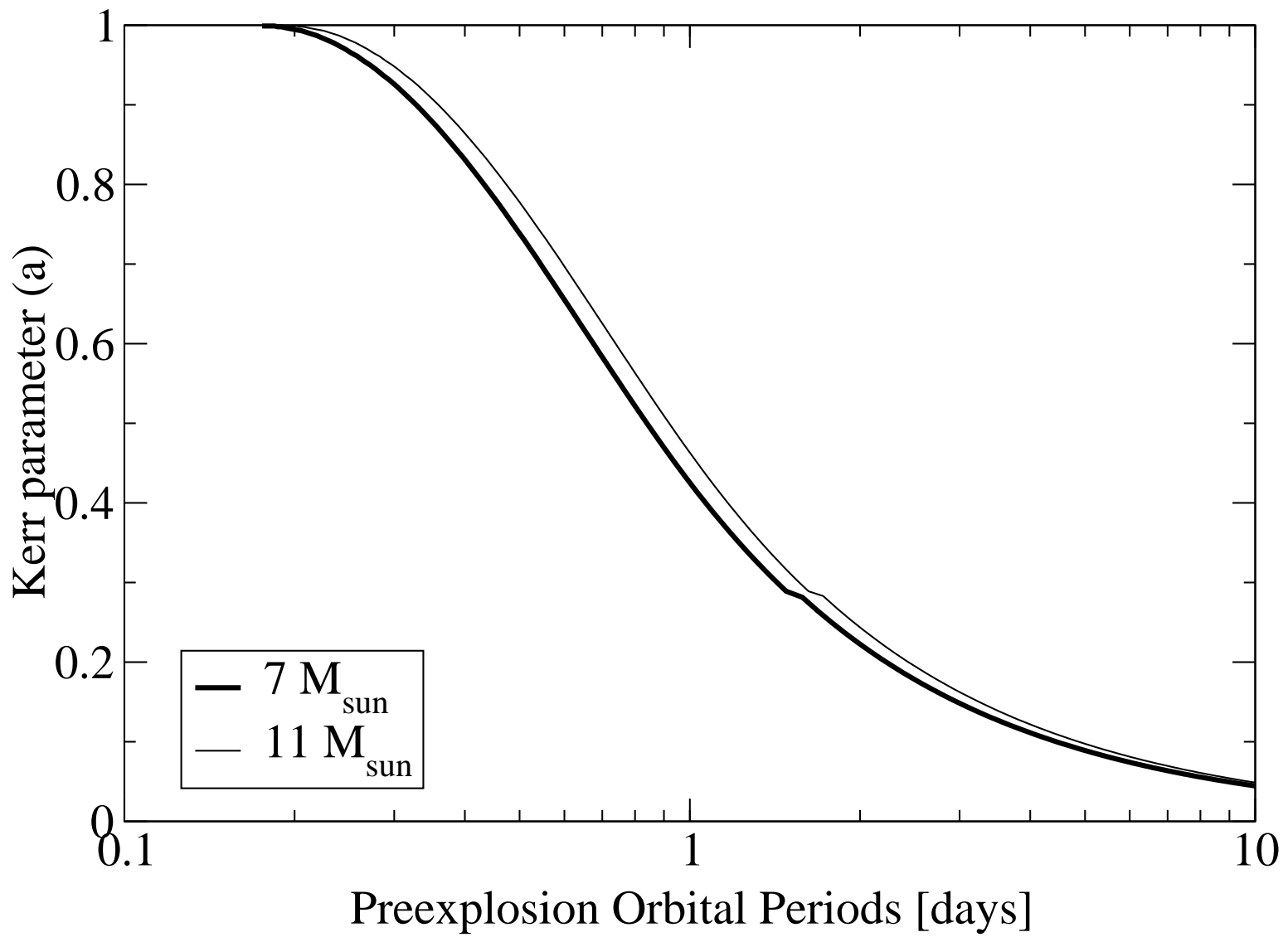

FIG. 1. - The Kerr parameter of the black hole resulting from the collapse of a helium star synchronous with the orbit, as a function of orbital period (LBW). Note that the result depends very little on the mass of the helium star, or on whether we use a simple polytrope or a more sophisticated model. 